\title{
An Intron 7 Polymorphism in APP Affects the Age of Onset of Dementia in Down Syndrome
}

\author{
Emma L. Jones, ${ }^{1}$ Clive G. Ballard, ${ }^{1}$ Vee P. Prasher, ${ }^{1}$ Matthew Arno, ${ }^{2}$ Stephen Tyrer, ${ }^{3}$ \\ Brian Moore, ${ }^{3}$ and Maria Luisa Hanney ${ }^{4}$ \\ ${ }^{1}$ Wolfson Centre for Age-Related Diseases, Guy's Campus, King's College London, London SE1 1UL, UK \\ ${ }^{2}$ Genomics Centre, Franklin-Wilkins Building, King's College London, London SE1 9NH, UK \\ ${ }^{3}$ Department of Psychiatry, Royal Victoria Infirmary, Newcastle Upon Tyne NE1 4LP, UK \\ ${ }^{4}$ Learning Disability Medical Directorate, Northgate Hospital, Morpeth, Northumberland NE61 3PB, UK \\ Correspondence should be addressed to Emma L. Jones, emma.3.jones@kcl.ac.uk
}

Received 23 August 2010; Revised 21 October 2010; Accepted 6 December 2010

Academic Editor: Lindsay A. Farrer

Copyright (๑) 2011 Emma L. Jones et al. This is an open access article distributed under the Creative Commons Attribution License, which permits unrestricted use, distribution, and reproduction in any medium, provided the original work is properly cited.

\begin{abstract}
People with Down syndrome (DS) develop Alzheimer's disease (AD) with an early age of onset. A tetranucleotide repeat, attt ${ }_{5-8}$, in intron 7 of the amyloid precursor protein has been associated with the age of onset of AD in DS in a preliminary study. The authors examine the impact of this polymorphism in a larger cohort of individuals with DS. Adults with DS were genotyped for attt ${ }_{5-8}$ and $A P O E$. The results were analysed with respect to the age of onset of dementia. The presence of three copies of the six-repeat allele resulted in onset of dementia seven years earlier than in the presence of other genotypes. Further study is essential to elucidate the mechanism by which this polymorphism functions, with an exciting opportunity to identify novel treatment targets relevant for people with DS and AD.
\end{abstract}

\section{Introduction}

Down syndrome (DS), defined cytogenetically by trisomy 21 , is the most common chromosomal disorder associated with learning disability, occurring in approximately $1 / 1000$ live births [1]. The prevalence of Alzheimer's disease (AD) in people with DS increases significantly with age. However, the nature of the early clinical presentation, course, and incidence rates of dementia are uncertain. Despite the nearly universal occurrence of $\mathrm{AD}$ pathology by age $40[2,3]$, there is wide variation in age of onset of clinical dementia. Most studies have indicated that the average age at onset of dementia is between 50 and 55 years of age, with a range from 38-70 years [4]. Many studies have now confirmed that age-related cognitive decline and dementia affecting people with DS occurs 30-40 years earlier than in the general population. Age-specific rates of dementia begin to increase in the patient's 30 s from $1-2 \%$ to $40 \%$ in the 50 s [5], hence dementia is becoming an increasingly important issue in people with DS as life expectancy continues to increase.
The neuropathological manifestations of $\mathrm{AD}$ in $\mathrm{DS}$ have been at least in part attributed to triplication and overexpression of the gene for amyloid precursor protein $(A P P)$ located on chromosome 21 [6]. In fact, an additional copy of $A P P$ can cause early onset AD with cerebral amyloid angiopathy, even when only small regions of chromosome 21 including only the APP gene are triplicated [7-10]. Triplication of chromosome 21 leads to an increase in expression levels of its genes. APP is expressed at levels which are four- to fivefold higher in DS than in the general population [11]. This is not only due to triplication of the gene, but is also caused by regulators of APP expression, for example ETS2, present on chromosome 21, which have increased expression levels [12]. Processing of APP can result in the production of betaamyloid $(\mathrm{A} \beta)$ which is deposited extracellularly as a core disease feature in the brains of people with AD.

Along with overexpression, other APP changes are seen in $\mathrm{AD}$, which include variations in the proportion of APP splice variants. Three of these isoforms are APP695, APP751, and APP770. APP695 is predominantly expressed in neurons 
whilst APP751 and APP770 are ubiquitously expressed. A kunitz protease inhibitor domain (KPI) encoded by exon 7 is present in both APP751 and APP770, but not in APP695. The ratio of APP751: APP695 mRNA is found to be increased in the brains of people with $\mathrm{AD}[13,14]$, and there appears to be a relationship between this ratio and the density of plaques in the hippocampus and entorhinal cortex [15]. In fact, mice expressing human APP751, but not APP695, develop an ADlike pathology, which most closely resembles that found in the brains of young people with DS [16]. This pathology not only involves $\mathrm{A} \beta$, but also abnormal tau isoforms. Therefore, it would seem that not only overexpression of APP in DS, but also variation in isoforms, may have a role in determining the onset of AD.

Intron 7 of $A P P$ has been sequenced previously to determine if polymorphic sites which may have the potential to regulate exon 7 splicing were present [17]. The tetranucleotide repeat (attt $\left.{ }_{5-8}\right)$ was identified and the attt 6 allele was found to be the most common, present in $96 \%$ of the genotyped population. When this region was genotyped in a preliminary study of 105 people with DS, there was found to be an association between the number of attt ${ }_{6}$ alleles and the age of onset of dementia [18]. Specifically, those individuals carrying three copies of the attt ${ }_{6}$ allele developed dementia with an earlier age of onset than those with any other combination of the repeats.

In DS polymorphisms on chromosome 21 may have an amplified effect. As most people with DS develop AD with an early age of onset, these amplified genetic effects may allow us to more clearly detect the associated phenotypes, such as accelerated onset of dementia or severity. Such genetic effects may be too subtle to detect in the general population, but the mechanistic information provided will be valuable in drug design for all those at risk of developing AD. Following the preliminary study [18] we examine whether the tetranucleotide repeat, attt ${ }_{5-8}$, in intron 7 impacts upon age of onset of dementia in a larger cohort of individuals with DS.

\section{Materials and Methods}

2.1. Samples and Clinical Assessments. Clinical evaluation was completed and DNA samples were obtained from 291 adults with DS (Newcastle-181, Birmingham-78, MEADOWS Clinical Trial-32), substantially extending a previous pilot study [18]. In the previous study the difference in age of onset between the genotypes was 13.4 years, with a standard deviation of 7.2 years [18]. Using these values, a power calculation shows that a study using a total of 18 participants (9 attt $_{6}$ homozygotes and 9 heterozygotes) has a 95 percent chance to detect a difference at a two-sided 0.05 significance level.

This study was approved by the appropriate local research ethics committee and consent was obtained from the participants. Detailed prospective clinical assessments were completed which included the DAMES battery (Newcastle, MEADOWS) and the Adaptive Behaviour Scale (ABS) [19] (Birmingham, MEADOWS), which enabled the operationalized diagnosis of dementia by an expert in the field (MH, VP) according to the International Classification of Disease [20]. Clinicians were blind to genotyping results.

2.2. Genotyping. Blood samples were collected into $\mathrm{K}_{2}$ EDTA vacutainers (BD Diagnostics), and DNA was extracted using the FlexiGene DNA kit (Qiagen). Genotyping of the $A P P$ intron 7 tetranucleotide repeat was performed using a CEQ8000 Genetic Analyser (Beckman Coulter). Briefly, the region surrounding the repeat was amplified by PCR (Dye 4-labelled forward primer 5'CCATGTCGTTAACAGACTTCC 3', reverse primer 5'GAGTAGTTCATACTTCCTAC3', both from Proligo). PCR enzymes and buffers were from Qiagen. Cycling was carried out as follows: $94^{\circ} \mathrm{C}$ for 2 minutes, 35 cycles of $\left(94^{\circ} \mathrm{C}\right.$ for 30 seconds, $55^{\circ} \mathrm{C}$ for 30 seconds, $72^{\circ} \mathrm{C}$ for 1 minute), $72^{\circ} \mathrm{C}$ for 10 minutes. This reaction produced a $105 \mathrm{bp}$ product for the attt ${ }_{6}$ allele. Labelled PCR products were combined with a $400 \mathrm{bp}$ size standard and sample loading solution (Beckman Coulter) and processed on the CEQ8000 genetic analyser.

$A P O E$ genotyping was carried out using PCR (for-

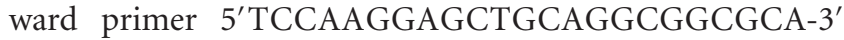
and reverse primer $5^{\prime}$-ACAGAATTCGCCCCGGCCTGGTACACTGCCA-3', Sigma-Aldrich). AmpliTaq Gold enzyme and buffer (Applied Biosystems) and 10\% DMSO (SigmaAldrich) were used, and cycling proceeded as follows: $94^{\circ} \mathrm{C}$ for 5 minutes, 45 cycles of $94^{\circ} \mathrm{C}$ for 30 seconds, $65^{\circ} \mathrm{C}$ for 1 minute, $72^{\circ} \mathrm{C}$ for 1 minute, and $72^{\circ} \mathrm{C}$ for 10 minutes. The resulting product was digested with HaeII or AflIII (New England Biolabs). Fragments were separated on 1\% standard agarose (Invitrogen Life Technologies) gels containing 3\% Metaphor agarose (Cambrex).

2.3. Statistics. Statistical analysis of clinical and genotyping data was carried out using SPSS version 17. For the primary analysis we compared the age of onset of dementia in people with and without the attt ${ }_{6}$ polymorphism using independent sample $t$-tests. The same evaluation was used to examine the impact of $A P O E-\varepsilon 4$.

\section{Results}

Two-hundred and ninety-one DNA samples were genotyped for the intron 7 repeat polymorphism attt $5_{-8}$ in $A P P$ and $A P O E$, using fragment analysis with a CEQ8000 genetic analyser and restriction fragment length polymorphism, respectively. Clinical data for each participant was collected by the examining psychiatrists. The mean age of the participants was 52.20 years \pm 13.93 (range from 24 to 89 years), and $38 \%$ were female. Based upon the operationalized psychiatric evaluations, 103 participants had dementia (35\%), with a mean age of onset of 50.46 years \pm 7.85 (range $34-74$ years). A further 76 individuals were 45 or older, but did not meet operationalized criteria for a diagnosis of dementia. Thirty people $(10.3 \%)$ were heterozygous for the attt $t_{-8}$ genotype, which is in agreement with frequencies found in previous genotyping studies $[17,18]$. Of the 103 participants with dementia, 9 were heterozygous for the tetranucleotide repeat polymorphism, which is an appropriately sized sample when 
considering the power calculation shown in Section 2. Due to variation in the peak sizes for the fragment analysis it is not possible to tell if the heterozygotes have one or two copies of the rarer alleles, so we have chosen to group all heterozygotes together based upon the presence of alleles attt 5 , attt 7 , or attt $_{8}$. This may be due to variations in amplification efficiencies between the different repeat alleles.

A significant difference was evident in the age of onset of dementia between individuals with 3 attt $_{6}$ alleles and those with any other allele combination $(n=103$, independent samples $t$-test, $t=-2.65$, df $=102, P=.009$ ) (a KaplanMeier plot is shown in Figure 1). The mean age of onset in individuals with 3 attt $_{6}$ alleles was 49.85 years \pm 7.81 whilst in people with a different repeat combination the mean age was 56.89 years \pm 5.09 . None of the individuals with less than

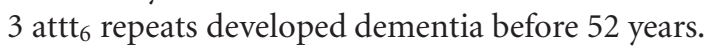

We also examined the link between the attt ${ }_{5-8}$ polymorphism and the risk of developing dementia before the age of 45. Only individuals who had dementia or had reached the age of 45 without developing dementia were included in this analysis, as individuals under the age of 45 and without dementia have not yet reached the high-risk period for dementia development. A trend was found between the risk of developing dementia before the age of 45 and the APP polymorphism $\left(n=179, \chi^{2}=2.95\right.$, df $\left.=1, P=.086\right)$.

Gender was not related to the age of onset of dementia $(t=-1.19, \mathrm{df}=84, P=.24)$. The presence of an $A P O E-\varepsilon 4$ allele was not associated with the age of onset of dementia $(t=0.49, \mathrm{df}=80, P=.62)$. Neither gender nor the presence of an $A P O E-\varepsilon 4$ allele were associated with attt $5-8$ genotype $\left(\chi^{2}=1.22, \mathrm{df}=1, P=.27\right.$ and $\chi^{2}=0.27, \mathrm{df}=1, P=.60$, resp.).

\section{Discussion}

These data highlight a striking association between 3 copies of the $a_{t t t}{ }_{6}$ allele in intron 7 of $A P P$ and a substantially earlier age of onset of dementia. People with 3 attt $_{6}$ alleles developed dementia 7 years earlier. The next step is to determine how the acceleration of dementia in DS is brought about in those people with 3 attt $_{6}$ alleles and to understand the implications for $\mathrm{AD}$ in the wider population, so that the related novel therapeutic opportunities can be fully utilized.

The tetranucleotide repeat region is located $1200 \mathrm{bp}$ into the $2598 \mathrm{bp}$ sequence of intron 7 and is $193 \mathrm{bp} 3^{\prime}$ from an Alu sequence which is key for binding of splicing factors and splicing of exons 7 and 8 from APP [7, 21] (Figure 2). The tetranucleotide repeat lies in a 524 bp region between single nucleotide polymorphisms (SNPs) rs9982544 and rs2409162, but neither of these is polymorphic in the CEU population and so cannot be used for haplotype analysis of this region for our samples. The closest SNPs which are sufficiently polymorphic in the CEU population, rs3787637 and rs8132200, enclose a region of 2313 bp extending beyond intron 7, and further analysis of how the tetranucleotide repeat is associated with these will be required before we can understand the linkage of this location.

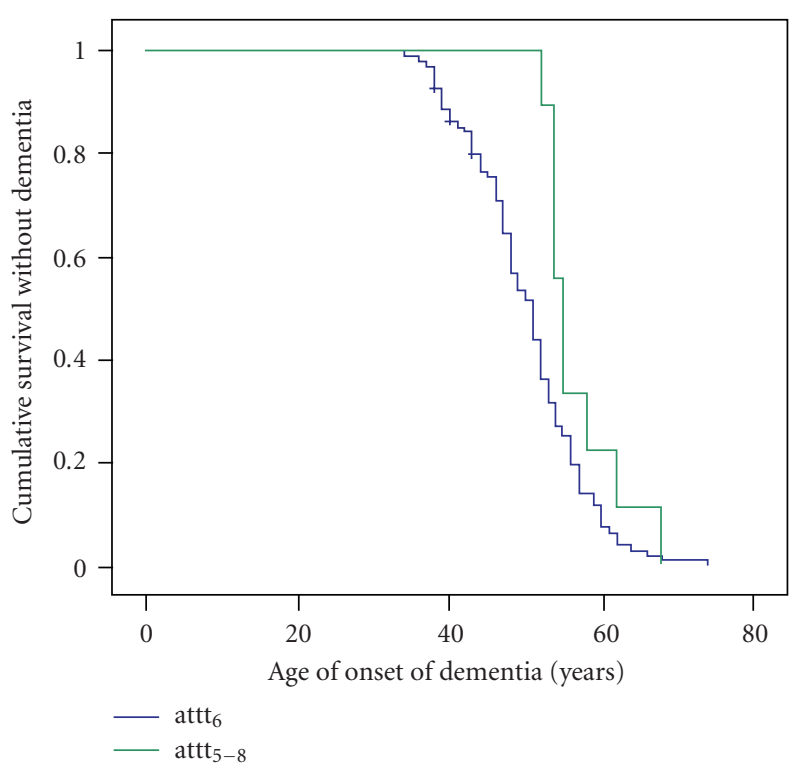

Figure 1: Kaplan-Meier survival plot showing the effect of the tetranucleotide attt $_{5-8}$ upon age of onset of dementia in 103 people with DS. Those people carrying three copies of the $\mathrm{attt}_{6}$ allele of this polymorphism develop dementia with an earlier age of onset than those with any other combination of alleles $(P=.009)$.

It is unclear why the 6-repeat allele is associated with risk when neither the 5- or 7-repeat alleles are, and why risk is not associated with increasing or decreasing repeat length although similar results have been found for other genes and disease risk: CYP19 and breast cancer risk, and MIF and gastric cancer risk $[22,23]$.

A further possibility is that the tetranucleotide repeat attt $_{6}$ is a marker for a functional polymorphism which is altering the expression, activity, or regulation of APP, which in turn alters the development of pathology and onset of dementia. Further examination of other SNPs in APP and how they are linked to attt $_{6}$ is required to understand fully the mechanism involved.

Levels of exon 7-containing isoforms of APP are found to be increased in AD brain [10] and associated with increased deposits of $\mathrm{A} \beta$ [24]. The protease inhibitor activity of the KPI domain has been shown to act on an extracellular trypsinlike serine protease which degrades secreted APP [25] and therefore, isoform variation may play an important role in regulating the activity of this protease. KPI-containing APP may also bind to low-density lipoprotein receptor-related protein (LRP), which is involved in the clearance of $A \beta$ from the brain into peripheral tissues, and this has been shown to reduce $A \beta$ clearance [26]. Receptors and other proteins involved in this clearance process may prove to be useful drug targets, and early intervention in this pathway may provide a method of preventing increased deposition of $A \beta$ in the brain.

The role of this polymorphism, or other associated polymorphisms, in regulation of APP splicing is not known, and examining this will play an important role in determining how dementia risk is affected. It will also be important to 


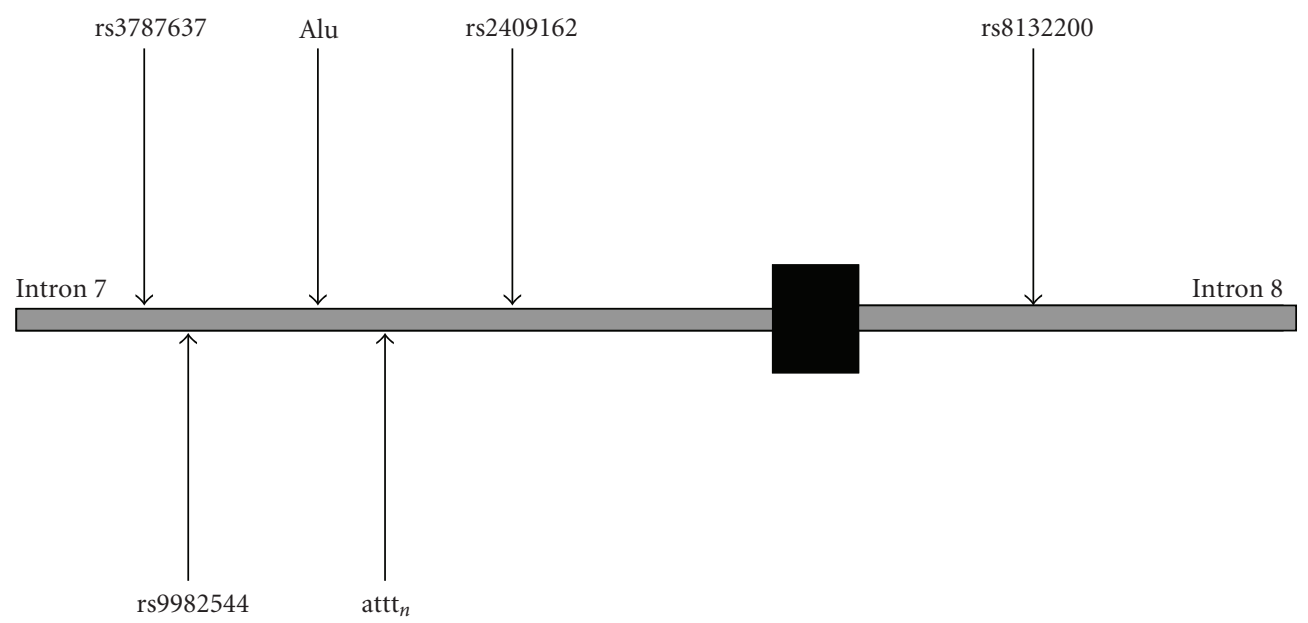

FIGURE 2: Diagrammatic representation of introns 7 to 8 of $A P P$ showing the locations of the tetranucleotide repeat, Alu sequence and closest SNPs. Grey bars indicate introns and black bar indicates exon 8. Figure is not to scale.

determine the impact of the tetranucleotide repeat upon $\mathrm{A} \beta$ deposition in the brain in postmortem studies. This will give an indication of how large a role this polymorphism plays in $\mathrm{A} \beta$ regulation.

The APOE- $\varepsilon 4$ allele had no effect upon the age of onset of dementia in this study. Previously it has been suggested that APOE does not affect the age of onset of dementia in cases of familial Alzheimer's disease (FAD) due to chromosome 14 mutations [27]. It may be that when APP regulation is strongly affected through triplication in DS or through particular mutations, APOE effects are masked by the presence of the more powerful APP effects. Triplication of APP in DS may have resulted in amplified effects of this polymorphism, but it may still have important potential implications for $\mathrm{AD}$ which have not yet been detected in the general population.

When this polymorphism was previously studied by Li et al. [17] the less common repeat sizes were only found in a small number of samples, which may result in low statistical power. Given that this polymorphism appears to take effect in a population with an increased risk of $\mathrm{AD}$ and increased expression of APP leading to increased A $\beta$ levels, it would be valuable to examine the effects of this polymorphism in a second population with an increased incidence of $\mathrm{AD}$ and $\mathrm{A} \beta$ concentrations, namely those in the wider population over the age of 80 . The incidence of $\mathrm{AD}$ in this age group is approximately $25 \%$, which is similar to that for people with DS in their 40s, and those over the age of 80 also have increased brain A $\beta 1-40$ and 1-42 levels [28]. A large study in such a population would allow us to analyse the effects of this attt $_{5-8}$ in the general population at risk of developing AD.

In this study we have taken a unique opportunity to examine AD-type dementia in a population in which a high proportion of people will develop dementia with an early age of onset. This has allowed us to examine more closely the association of this polymorphism to the development and progression of dementia. The amplification of the effects of $A P P$ polymorphisms in DS allows us to detect differences which may be more subtle in the wider population.
Studies such as these not only provide the opportunity to discover therapies which may benefit those with DS, but also those in the older general population in which the incidence of $\mathrm{AD}$ is increasing. The benefits of studying the relatively small DS population must not be forgotten in the search for new therapies to tackle AD.

\section{Conclusions}

The attt $5-8$ polymorphism in $A P P$ is associated with the age of onset of $\mathrm{AD}$ in $\mathrm{DS}$, from which we conclude that it may function by accelerating the pathological process or is a marker for an, as yet unidentified, functional variant. The mechanism by which this is achieved has yet to be established, but the proteins involved may prove to be useful targets for future drug development.

\section{Conflict of Interests}

The authors report no conflict of interests.

\section{Acknowledgments}

The authors would like to thank the individuals who participated in this study and their families, and the Alzheimer's Research Trust for funding the study. The authors would also like to acknowledge Dr. Chris Morris of the Institute for Ageing and Health, Newcastle General Hospital for his discussions.

\section{References}

[1] E. B. Hook, "Rates and Down's syndrome in live births and at midtrimester amniocentesis," Lancet, vol. 1, no. 8072, pp. 1053-1054, 1978.

[2] H. M. Wisniewski, W. Silverman, and J. Wegiel, "Ageing, Alzheimer disease and mental retardation," Journal of Intellectual Disability Research, vol. 38, no. 3, pp. 233-239, 1994. 
[3] R. D. Terry, L. A. Hansen, and R. DeTeresa, "Senile dementia of the Alzheimer type without neocortical neurofibrillary tangles," Journal of Neuropathology and Experimental Neurology, vol. 46, no. 3, pp. 262-268, 1987.

[4] V. P. Prasher and V. H. R. Krishnan, "Age of onset and duration of dementia in people with Down syndrome: integration of 98 reported cases in the literature," International Journal of Geriatric Psychiatry, vol. 8, no. 11, pp. 915-922, 1993.

[5] A. J. Holland, J. Hon, F. A. Huppert, F. Stevens, and P. Watson, "Population-based study of the prevalence and presentation of dementia in adults with Down's syndrome," British Journal of Psychiatry, vol. 172, pp. 493-498, 1998.

[6] B. Rumble, R. Retallack, C. Hilbich et al., "Amyloid A4 protein and its precursor in Down's syndrome and Alzheimer's disease," New England Journal of Medicine, vol. 320, no. 22, pp. 1446-1452, 1989.

[7] A. Rovelet-Lecrux, D. Hannequin, G. Raux et al., "APP locus duplication causes autosomal dominant early-onset Alzheimer disease with cerebral amyloid angiopathy," Nature Genetics, vol. 38, no. 1, pp. 24-26, 2006.

[8] K. Sleegers, N. Brouwers, I. Gijselinck et al., "APP duplication is sufficient to cause early onset Alzheimer's dementia with cerebral amyloid angiopathy," Brain, vol. 129, no. 11, pp. 2977-2983, 2006.

[9] L. Cabrejo, L. Guyant-Maréchal, A. Laquerrière et al., "Phenotype associated with APP duplication in five families," Brain, vol. 129, no. 11, pp. 2966-2976, 2006.

[10] K. Kasuga, T. Shimohata, A. Nishimura et al., "Identification of independent APP locus duplication in Japanese patients with early-onset Alzheimer disease," Journal of Neurology, Neurosurgery and Psychiatry, vol. 80, no. 9, pp. 1050-1052, 2009.

[11] K. Beyreuther, P. Pollwein, G. Multhaup et al., "Regulation and expression of the Alzheimer's $\beta / A 4$ amyloid protein precursor in health, disease, and Down's syndrome," Annals of the New York Academy of Sciences, vol. 695, pp. 91-102, 1993.

[12] E. W. Wolvetang, O. M. Bradfield, M. Tymms et al., "The chromosome 21 transcription factor ETS2 transactivates the $\beta$ APP promoter: implications for Down syndrome," Biochimica et Biophysica Acta - Gene Structure and Expression, vol. 1628, no. 2, pp. 105-110, 2003.

[13] R. E. Tanzi, A. I. McClatchey, E. D. Lamperti, L. VillaKomaroff, J. F. Gusella, and R. L. Neve, "Protease inhibitor domain encoded by an amyloid protein precursor mRNA associated with Alzheimer's disease," Nature, vol. 331, no. 6156, pp. 528-530, 1988.

[14] T. Matsui, M. Ingelsson, H. Fukumoto et al., "Expression of APP pathway mRNAs and proteins in Alzheimer's disease," Brain Research, vol. 1161, no. 1, pp. 116-123, 2007.

[15] S. A. Johnson, T. McNeill, B. Cordell, and C. B. Finch, "Relation of neuronal APP-751/APP-695 mRNA ratio and neuritic plaque density in Alzheimer's disease," Science, vol. 248, no. 4957, pp. 854-857, 1990.

[16] L. S. Higgins, R. Catalano, D. Quon, and B. Cordell, "Transgenic mice expressing human $\beta$-APP751, but not mice expressing $\beta$-APP695, display early Alzheimer's disease-like histopathology," Annals of the New York Academy of Sciences, vol. 695, pp. 224-227, 1993.

[17] L. Li, R. Perry, J. Wu et al., "Polymorphic tetranucleotide repeat site within intron 7 of the $\beta$-amyloid precursor protein gene and its lack of association with Alzheimer's disease," Human Genetics, vol. 103, no. 1, pp. 86-89, 1998.

[18] M. Margallo-Lana, C. M. Morris, A. M. Gibson et al., "Influence of the amyloid precursor protein locus on dementia in Down syndrome," Neurology, vol. 62, no. 11, pp. 1996-1998, 2004.

[19] V. Prasher, A. Farooq, and R. Holder, "The Adaptive Behaviour Dementia Questionnaire (ABDQ): screening questionnaire for dementia in Alzheimer's disease in adults with Down syndrome," Research in Developmental Disabilities, vol. 25, no. 4, pp. 385-397, 2004.

[20] World Health Organisation, International Classification of Diseases, WHO, Geneva, Switzerland, 10th edition, 1992.

[21] R. Donev, A. Newall, J. Thome, and D. Sheer, "A role for SC35 and hnRNPA1 in the determination of amyloid precursor protein isoforms," Molecular Psychiatry, vol. 12, no. 7, pp. 681690, 2007.

[22] C. A. Haiman, S. E. Hankinson, D. Spiegelman et al., "A tetranucleotide repeat polymorphism in CYP19 and breast cancer risk," International Journal of Cancer, vol. 87, no. 2, pp. 204210, 2000.

[23] H. Shiroeda, T. Tahara, T. Shibata et al., "Functional promoter polymorphisms of macrophage migration inhibitory factor in peptic ulcer diseases," International Journal of Molecular Medicine, vol. 26, no. 5, pp. 707-711, 2010.

[24] M. Barrachina, E. Dalfó, B. Puig et al., "Amyloid- $\beta$ deposition in the cerebral cortex in Dementia with Lewy bodies is accompanied by a relative increase in A $\beta P P$ mRNA isoforms containing the Kunitz protease inhibitor," Neurochemistry International, vol. 46, no. 3, pp. 253-260, 2005.

[25] M. D. Caswell, SU. S. Mok, A. Henry et al., "The amyloid $\beta$-protein precursor of Alzheimer's disease is degraded extracellularly by a Kunitz protease inhibitor domain-sensitive trypsin-like serine protease in cultures of chick sympathetic neurons," European Journal of Biochemistry, vol. 266, no. 2, pp. 509-516, 1999.

[26] R. D. Moir and R. E. Tanzi, "LRP-mediated clearance of $\mathrm{A} \beta$ is inhibited by KPI-containing isoforms of APP," Current Alzheimer Research, vol. 2, no. 2, pp. 269-273, 2005.

[27] C. Van Broeckhoven, H. Backhovens, M. Cruts et al., "APOE genotype does not modulate age of onset in families with chromosome 14 encoded Alzheimer's disease," Neuroscience Letters, vol. 169, no. 1-2, pp. 179-180, 1994.

[28] H. Lewis, D. Beher, N. Cookson et al., "Quantification of Alzheimer pathology in ageing and dementia: age-related accumulation of amyloid- $\beta(42)$ peptide in vascular dementia," Neuropathology and Applied Neurobiology, vol. 32, no. 2, pp. 103-118, 2006. 


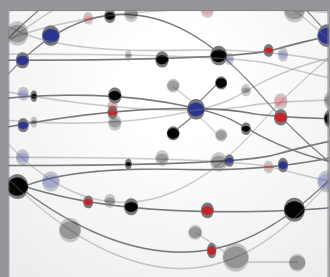

The Scientific World Journal
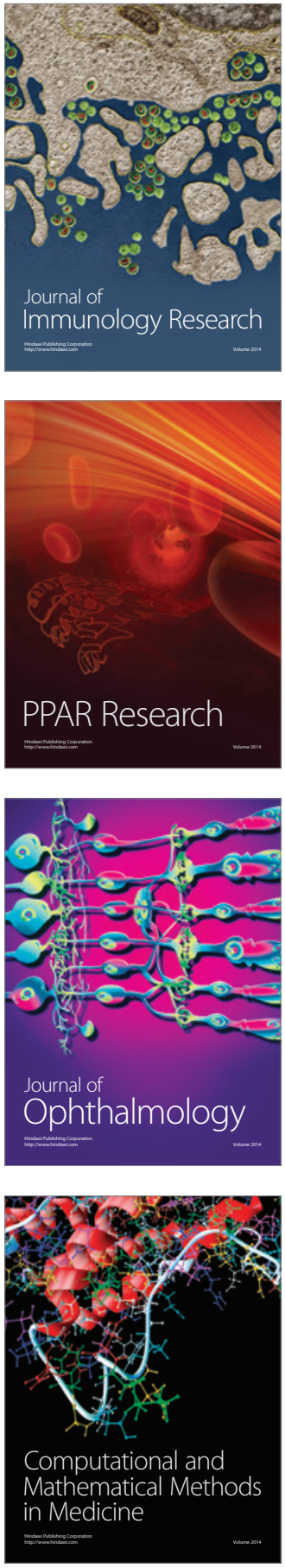

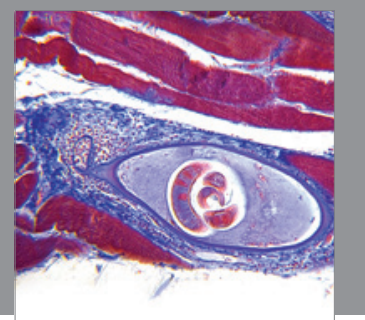

Gastroenterology

Research and Practice
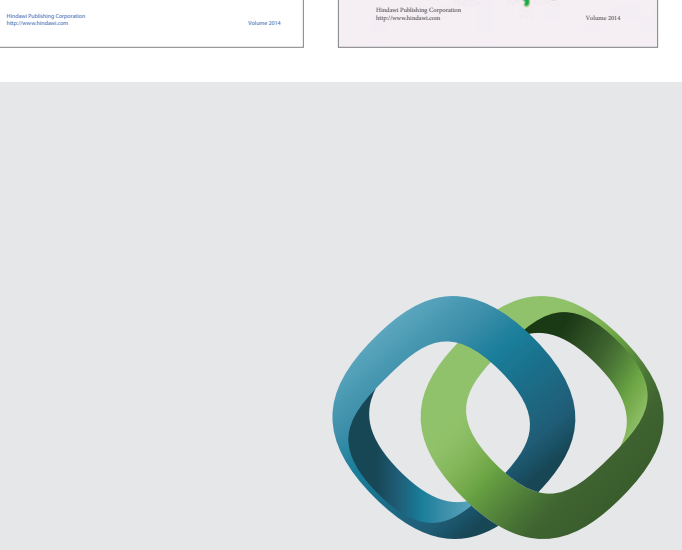

\section{Hindawi}

Submit your manuscripts at

http://www.hindawi.com
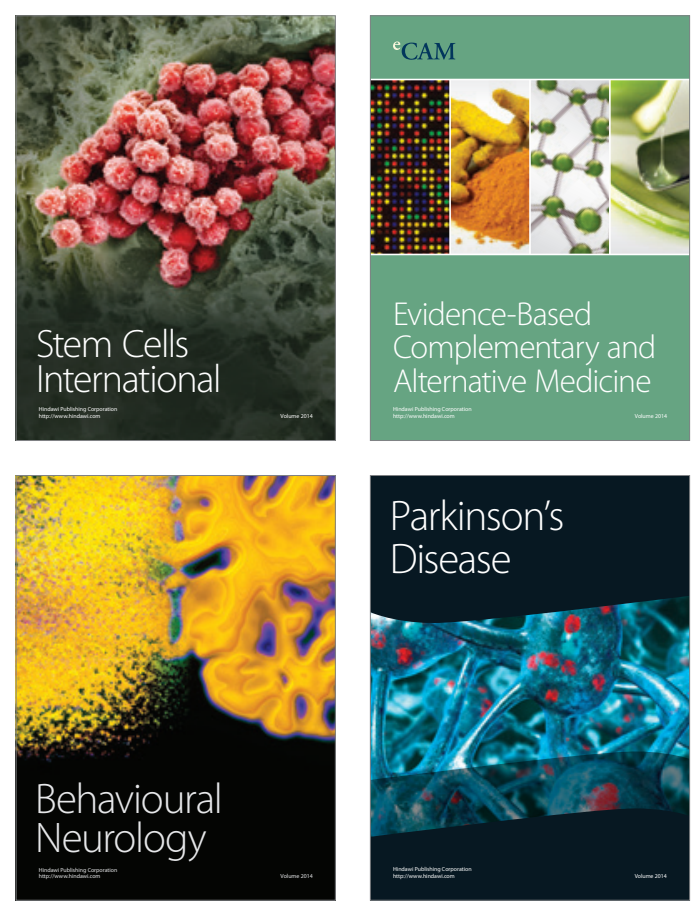

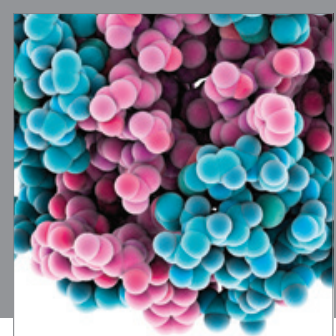

Journal of
Diabetes Research

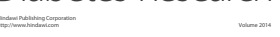

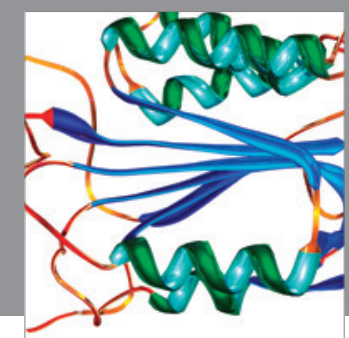

Disease Markers
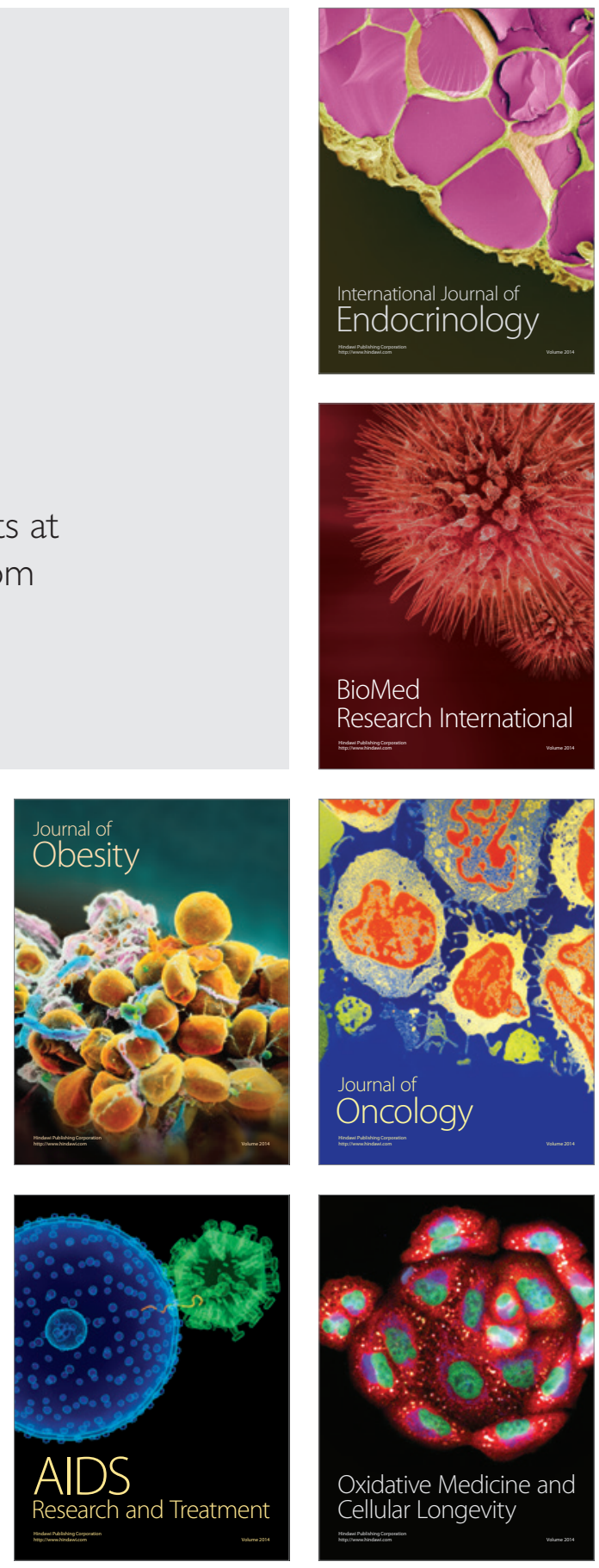